\title{
Comparación de un programa libre de drogas y uno de mantenimiento con metadona en adictos a opiáceos
}

\section{Comparison between a free drugs program with a methadone conservative program in opiates addicts}

José Miguel RODRÍGUEZ MOLINA*

\section{RESUMEN}

El problema del consumo de heroína no ha disminuido en los últimos años de forma significativa, pero los tratamientos aplicados no resultan demasiado eficaces. Eso ha hecho que se incremente el número de tratamientos con agonistas opiáceos. Sin embargo aquí se pone a prueba un programa libre de drogas (P.L.D.) basado en los principios de la modificación de conducta, con apoyo sociosanitario y el uso de antagonistas (naltrexona) durante la desintoxicación y durante el proceso de deshabituación y se compara con un programa de mantenimiento con metadona (P.M.M). El P.L.D. Resulta ser superior en disminución de consumo de heroína, benzodiacepinas y cocaina y en tasa de retención en tratamiento, no hallándose diferencias en anfetaminas y resulta inferior en consumo de cannabis. Se sugieren futuras líneas de investigación con otras variables dependientes de tipo psicológico y en P.M.M. con apoyo psicológico.

\section{PALABRAS CLAVE}

Opiáceos, Opioides, Heroína, Metadona, Naltrexona, Modificación de conducta.

\footnotetext{
* Departamento de Psicología Biológica y de la Salud. Universidad Autónoma de Madrid.
} 
Comparación de un programa libre de drogas y uno de mantenimiento con metadona en adictos a opiáceos

\section{ABSTRACT}

The problem of heroine consumption hasn't decrease significantly in latest years, but the applied treatments are not effective enough. That made increase the number of treatments with opiates agonists. However, we test here a free drugs program (FDP) based on principles of modifying behaviour with health and social support and the use of antagonists (naltrexone) during disposing and also during the disaccustom process and we compare it with a methadone conservative program (MCP). The FDDP worked better in decreasing heroine, benzodiazepines and cocaine consumption and in retention rate during treatment, there were no differences in amphetamines and it worked less in cannabis consumption. We suggest future ways of research with other variables depending of psychological type and in MCP with psychological support.

\section{KEY WORDS}

Opiates, Opioids, Heroine, Methadone, Methadone, Behavior modification. 
El consumo habitual de heroína es relevante epidemiológicamente en España desde los años 80 (Comas, 1.993). En los 90, por la aparición del SIDA, el consumo comienza a cambiar de vía, de parenteral a nasal.

Al tratarse de una droga ilegal es dificil conocer la prevalencia. La mayor parte de los estudios dan cifras en torno al $0,1 \%$ de la población adulta, pero Granfield y Cloud, (1.996) estiman un alto porcentaje de "población escondida" que no aparece en las estadisticas. En todo caso el porcentaje de tratamientos que se realizan por consumo de heroina se mantiene en torno al $70 \%$ de los tratamientos totales.

\section{DEFINICIÓN DEL PROBLEMA}

Podemos afirmar que existen dos mecanismos biológicos que explican el consumo de drogas: $\mathrm{O}$ bien se consumen drogas porque éstas activan circuitos de recompensa cerebral, o porque una vez que es habitual el consumo, su ausencia produce alteraciones fisiológicas desagradables que denominamos sindrome de abstinencia (S.A.O. en el caso de opiáceos). Desde un punto de vista psicológico, decimos que el sujeto consume heroína para producirse estados placenteros 0 para evitar el S.A.O., o sea, por procedimientos de reforzamiento positivo 0 negativo (Graña, 1994).

Las consecuencias negativas de la adicción a la heroína son múltiples: enfermedades como la infección por los virus de la hepatitis y el VIH (Caballo y cols., 1.990; Buzón, 1.995), debido fundamentalmente al intercambio de jeringuillas, así como otras como las producidas por Cándidas o la tuberculosis. Además, según Flynn y cols., (1.998) en un estudio con 7.402 pacientes, un $40 \%$ presentaba psicopatologia. Hay que aña- dir además otros problemas como el riesgo de sobredosis, los problemas sociales (familiares, laborales, culturales, delictivos,...).

\section{TRATAMIENTOS}

No existe un tratamiento de eficacia absoluta ni desde el punto de vista de los resultados (Nahas y Trouvé, 1.992), ni del de la demanda (Plan Nacional Sobre Drogas, 1.995): Muchos sujetos recaen y otros ni siquiera acuden a tratamiento.

Los bajos resultados que presentan los Programas Libres de Drogas (P.L.D.), han producido un auge de los programas de reducción del daño, cuyo objetivo ya no es el cese del consumo sino otros, como la sustitución de la sustancia por otra menos dañina, o la reducción de los daños (como es el caso de los programas de intercambios de jeringuillas). Destacan entre ellos los Programas de Mantenimiento con Metadona (P.M.M.). Podemos hacer, por lo tanto, una gran diferenciación entre dos tipos de programas: Los de mantenimiento con agonistas y los libres de drogas.

La Metadona es un agonista opiáceo sintético, que produce efectos similares a la morfina pero que carece de los efectos psicológicos reforzantes de la heroína (Torres Hernández y García Barrachina, 1.997).

El uso de los P.M.M. se basa en la idea de que hay pacientes que no pueden permanecer abstinentes a opiáceos por problemas orgánicos, por lo que precisan el aporte de cantidades suplementarias de opiáceos (Duro, Casas y Colom, 1.994), o bien, que no quieren dejar el consumo de opiáceos y se les puede ofrecer una alternativa de consumo controlado. El alarmante incremento de los casos de VIH, hizo que en la década de 
los 90 se comenzaran a implantar progresivamente los P.M.M. (Bobes-García y cols., 1.993). Los criterios para la prescripción de Metadona varían de unos planes a otros, pero se ha ido haciendo más laxos, llegando a darse "a demanda".

Los datos respecto a su efectividad son contradictorios. Así, Ball y Ross (1.991), informan de una abstinencia total en el $92 \%$ de los sujetos del P.M.M. lo que contrasta con lo hallado en este estudio. Escudero i Torras y Ferrer i Pérez (1.996) informan de una retención media en el P.M.M. del 83,8\%. y Pascual Fernández (1.999) encontró una tasa de retención del $92,7 \%$ a los doce meses.

Sin embargo, Marlatt y cols. (1.997) afirma que los programas de reducción del daño son útiles pero porque "pueden facilitar la entrada" a los programas de tratamiento libres de drogas. El otro gran tipo de tratamiento, el P.L.D., tiene por objetivos el cese del consumo de drogas, así como la producción de los cambios necesarios (biológicos, psicológicos y sociales) para mantener la abstinencia.

En contra de lo que pudiera parecer, por el incremento de los P.M.M., la demanda de P.L.D. no se reduce significativamente (Agencia Antidroga, 2.004).

Frecuentemente, los pacientes con dependencia a la heroina, que optan por un P.L.D., necesitan una desintoxicación previa, hospitalaria o ambulatoria. En el caso más exitoso en la red pública, las desintoxicaciones hospitalarias, el éxito oscila entre el 70\% y el 80\% (Agencia Antidroga, 2000).

El objetivo del P.L.D. tras la desintoxicación, debe ser el cambio de patrones cognitivos y conductuales y en general de su estilo de vida, sustituyéndolo por uno más saludable (Plan Regional Sobre
Drogas, 1.995), así como la adquisición de nuevas habilidades de afrontamiento que le permitan "vivir satisfecho consigo mismo y la sociedad y hacer frente a los problemas diarios sin recurrir a las drogas” (Nahas y Trouvé, 1.992).

Hay que diferenciar el fallo ocasional ("lapse") de la recaída ("relapse") (Marlatt, 1.985-a y d). En esta linea, Kelley (1.989), considera que la retención es un indice aceptable de éxito. Pero las tasas de retención suelen ser históricamente muy bajas. Desde el estudio pionero de Hunt y cols., (1.971), aparecen tasas de retención anual de solamente el 30\%, la misma cifra que encontraron quince años después Maddux y Desmond (1.986). El Proyecto EMETYST, encontró que las altas por fin de tratamiento eran solo del 4\% (Plan Nacional sobre Drogas, 1.992) y el estudio DARP, realizado con más de 27.000 pacientes de 52 centros de Estados Unidos (Soler Insa, 1.981), encontró que tanto los P.L.D. como los P.M.M., tienen más efectividad que la ausencia de tratamiento, pero únicamente a condición de durar más de tres meses (muchos pacientes recaian antes). En un estudio en 1992 el Plan Nacional Sobre Drogas (1.992), calcula que la eficacia total es solo del 2,8\% anual. Las sucesivas memorias de la Comunidad de Madrid (Plan Regional Sobre Drogas, 1.992; Agencia Antidroga 1.999 a 2004) dan tasas de retención en los P.L.D. entre el 18 y el $25 \%$.

Pero hay datos mejores: Girón (1.996) en un su Tesis Doctoral, encuentra que el $51 \%$ se mantiene abstinente, seis años después de iniciar su tratamiento y en otro estudio (Ministerio de Sanidad y Consumo, 1.998), se halla una tasa de retención del $58,1 \%$.

Hay que hacer hincapié en que el consumo prolongado de opiáceos, produce un deterioro del sistema opioide, por 
neuroadaptación negativa (Self y Nestler 1.998). Aún estando desintoxicado, el sujeto tenderá a compensar ese déficit lanzando conductas de búsqueda de opiáceos (Casas y Salazar, 1993). Esta hipótesis es compatible con estas otras psicológicas: Schulteis, y Koob (1.996) opinan que el reforzamiento de la conducta abstinente y la neuroadaptación interactúan en el proceso de las recaídas, ya que el reforzamiento produce efectos dopaminérgicos que favorecen la neuroadaptación.

Además del malestar físico producido por la neuroadaptación negativa, son numerosos los factores que influyen en las recaídas (Marlatt, 1.978; 1.985-b, c y e; Marlatt y Gordon, 1.980; Cummings, Gordon y Marlatt, 1.980; Cummings, y cols., 1.980, Arce, 1.997 Llorente del Pozo, 1.997, Rodríguez Molina, 1.995, Kornblit y Mendes, 1.990; Bedate Villar, 1.998). Podemos agrupar en seis factores las causas de recaida: Los estados de ánimo negativos, el condicionamiento del deseo de consumo ("craving") o del S.A.O., los déficits de motivación, ciertas variables de tipo cognitivo, el entorno social y las habilidades para afrontarlo y por el malestar físico.

Por lo tanto una buena intervención en modificación de conducta debe tener en cuenta estos factores. El P.L.D. que ponemos a prueba, se enmarca en el modelo biopsicosocial, con uso de antagonista y de técnicas de modificación de conducta.

Las técnicas que conforman el P.L.D., son (García y Graña; 1.987; Echeburúa; 1.996): Uso de Antagonistas (Naltrexona), Red de apoyo social y control de estimulos, Apoyo y seguimiento de recursos sociales, Reestructuración cognitiva de las ideas de consumo y de depresión, Entrenamiento en habilidades sociales, Entrenamiento en técnicas de solución de problemas, Entrenamiento en técnicas de reducción de la ansiedad (relajación progresiva e inoculación al estrés), Estilo de vida sano, Motivación: reforzamiento y contrato terapéutico y Control de la abstinencia.

\section{OBJETIVOS}

El objetivo es comparar la eficacia de dos tratamientos para drogodependientes. Uno es un P.L.D. basado en los principios y técnicas de la Modificación de Conducta con naltrexona, y el otro es P.M.M.

A la hora de evaluar la bondad de un programa de tratamiento de drogodependencias hemos de valorar fundamentalmente dos aspectos: si los sujetos se mantienen en el programa y si mientras están en él consumen drogas ilegales. (Gutiérrez y cols., 1.996; Echeburúa 1.994)

\section{MÉTODO}

\section{Hipótesis:}

Se intentará probar la eficacia diferencial entre los dos programas para cada una de las variables dependientes, que se desarrollan más adelante. Esto nos da el siguiente conjunto de hipótesis:

H1: Los sujetos del P.L.D. tendrán menos proporción de controles toxicológicos positivos a opiáceos que los del P.M.M

H2: idem, a otras. Esta hipótesis se subdivide para cada sustancia no opiácea analizada, quedando así: H2-a: Los sujetos del P.L.D. tendrán menos proporción de controles positivos a Cocaína que los sujetos del grupo del P.M.M. , H2-b: Idem para Benzodiacepinas, H2-c: Idem 
para Anfetaminas y H2-d: Idem para Cannabis.

H3: Los sujetos drogodependientes en un P.L.D. tendrán una mayor tasa de retención en el Programa que los del grupo del P.M.M.

\section{Variables}

\section{Variables Dependientes}

La consecución de cada uno de los objetivos antes mencionados, da lugar a cada una de las variables dependientes. La primera variable dependiente (VD1) es el consumo de opiáceos, que se medirá a través de los registros de controles toxicológicos.

La segunda variable (VD2) es el consumo de otras sustancias, medida de igual forma que la anterior: VD2-a: consumo de Cocaína, VD2-b: consumo de Benzodiacepinas, VD2-c: consumo de Anfetaminas y VD2-d: consumo de cannabis.

La tercera variable (VD3) es la retención. Es la proporción entre sujetos que han recibido el Alta Terapéutica o permanecen en tratamiento y los que lo iniciaron.

\section{Variable Independiente:}

La variable independiente es el tratamiento, que consiste en la aplicación de un P.L.D. o de un P.M.M. Hay que recordar que existen otros P.M.M. en los que además del agonista, el sujeto recibe apoyo psicológico. No son objeto de este estudio dada la preeminencia de P.M.M. basados únicamente en la dispensación.

\section{Variables Extrañas y su control}

La aleatorización no es posible dado que se trató con pacientes reales: No se puede asignar a un sujeto al azar a un tratamiento libre de drogas o de metadona. Para controlar las variables extrañas se ha seguido el procedimiento de medición de las mismas (Martínez Hernández, 1.993 y 1.994).

No parece haber efecto de las otras fuentes clásicas de invalidez como la historia y la maduración, la situación de laboratorio, la medida previa y la regresión estadística. La pérdida de sujetos en nuestro caso esa es una variable dependiente.

\section{Sujetos}

Los sujetos del grupo de P.L.D. fueron seleccionados al azar de entre los de un centro privado especializado (CITA en Madrid), que permitió al autor desarrollar el P.L.D. De todos los sujetos a los que se pidió su participación en el programa, solo uno rechazó tal ofrecimiento. En total iniciaron el tratamiento cuarenta sujetos. Todos realizaron la desintoxicación U.R.O.D. (desintoxicación ultrarrápida a opiáceos, con naltrexona; Legarda y Gossop, 1994) y todos habían consumido heroina horas antes de la misma.

Los sujetos del Programa de Mantenimiento con Metadona (P.M.M) eran usuarios del Centro de Atención Integral a Drogodependientes de San Blas (Madrid). $\mathrm{Al}$ igual que en el Grupo del P.L.D., se iniciaron las mediciones con cuarenta sujetos. Hubo tres sujetos más que rechazaron participar en la investigación.

No hubo diferencias significativas entre los dos grupos en ninguna de las 
siguientes variables: sexo, edad, educación, situación laboral, años de consumo, cantidad de heroína consumida, problemas legales, vías de consumo, consumo habitual de cocaina, bezodiacepinas, anfetaminas y cannabis.

Sí hubo diferencias en la variable convivencia, en el grupo de P.L.D. los mismos sujetos vivian con sus padres o con su pareja, mientras que en el P.M.M. había tres veces más sujetos que vivian con sus padres. En el P.L.D. había un mayor número de trabajadores $(45,72 \%$ frente al 3,33\%). Todos los sujetos del grupo P.M.M. eran seropositivos al VIH, no constando este dato para los del P.L.D.

\section{Diseño}

Para probar cada una de las tres Hipótesis, Consumo de Opiáceos (H1); Consumo de Otras Sustancias (H2) y Retención en el Programa (H3) se realiza una comparación directa entre los dos grupos. En el caso de estas tres hipótesis no tiene sentido medir antes del tratamiento: Respecto al consumo de opiáceos (VD1), todos los sujetos estaban intoxicados antes del tratamiento, luego consumian a diario por definición. Respecto a otras drogas (VD2), no pueden tenerse datos fiables antes del tratamiento. La Retención (VD3), es una variable inherente al desarrollo del Programa.

La variable VD2, exige realizar una comparación igual para cada una de las subhipótesis que representan el consumo de Cocaina, Benzodiacepinas, Anfetaminas y Cannabis, respectivamente.

\section{Procedimiento}

A medida que en los dos centros de tratamiento aparecian personas que tuviesen los requisitos requeridos (en el caso del P.M.M. no realizar tratamiento psicológico) se pedía permiso a los sujetos para realizar el estudio con ellos y se les aplicaba el tratamiento correspondiente.

Para las variables VD1 y VD2, se halla cada proporción $\mathrm{P}$ de urinocontroles positivos: $\mathrm{P}=\left(\mathrm{n}^{\mathrm{0}}\right.$ controles positivos $/ \mathrm{n}^{\mathrm{0}}$ total de controles), para cada sujeto. La retención en el programa (VD3), se calculó como: $\mathrm{R}$ = (Sujetos que permanecen + finalizan) / Sujetos que inician.

Los sujetos del P.M.M. no realizaron ninguna desintoxicación y sustituyeron directamente la heroína por Metadona. Los sujetos del P.L.D. realizaron una desintoxicación U.R.O.D. y posteriormente se inició la administración de Naltrexona y el P.L.D. explicado. Este programa se desarrolla a través de sesiones de intervención individual con el psicólogo y en algún caso por el médico y el trabajador social. Además se realizan sesiones con la familia y amigos (Red de Apoyo Social).

\section{RESULTADOS}

Los datos fueron tratados con el programa SPSS/PC+. Los resultados, se muestran en los cuadros I y II.

En primer lugar es notable que el consumo de heroina (medido como proporción de controles positivos de todos los realizados para cada sujeto realizados) entre los pacientes que reciben otros opiáceos en su tratamiento es nueve veces mayor que entre los sujetos del P.L.D. Entre los primeros el 9\% de los controles resultaron positivos, frente a solo el $1 \%$ en el grupo de tratamiento.

Igualmente llamativo es que el número de positivos a cocaina fue notablemente mayor en el grupo del P.M.M. que en el 
P.L.D. El consumo de benzodiacepinas también fue mucho mayor en el grupo P.L.D. control, pero además el dato tiene relevancia clinica: uno de cada cinco controles a esta sustancia resultó positivo.

En ninguno de los dos grupos se detectó ningún consumo de anfetaminas y el grupo de P.L.D. dio más positivos a cannabis que el de P.M.M.

Otro dato muy significativo es que la retención a los seis meses fue un 12,5\% mayor en el P.L.D. que en el P.M.M. $(87,5 \%$ frente a $75 \%)$.

\section{CONCLUSIÓN}

Se rechaza la Hipótesis de igualdad de medias, para las variables dependientes:
VD1: Proporción de positivos a Opiáceos, VD2-a: Proporción de positivos a Cocaína, VD2-b: Proporción de positivos de Benzodiacepinas y VD3: Tasa de Retención.

Se verifica la Hipótesis Nula de igualdad de medias para las variables dependientes: VD2-c: Proporción de positivos a Anfetaminas y VD-d: Proporción de positivos a Cannabis.

\section{DISCUSIÓN}

El programa libre de drogas que se pone a prueba está formado por un conjunto de técnicas diferentes. Como ya se ha dicho no resulta posible aplicarlas por separado para conocer su efecto diferencial. De hecho, encontrar cuáles con los

Cuadro 1. Resumen de medidas y desviaciones tipicas de consumo de todas las sustancias (VD1 y VD2)

\begin{tabular}{|c|c|c|c|}
\hline \multirow{2}{*}{ SUSTANCIAS } & \multirow{2}{*}{ тто. } & \multicolumn{2}{|c|}{ PROPORCION DE POSITIVOS } \\
\hline & & MEDIA & D. T. \\
\hline OPIÁCEOS & \multirow{5}{*}{ P.M.M. } & 0.09 & 0.20 \\
\hline COCAÍNA & & 0.07 & 0.20 \\
\hline BENZODIACEPINAS & & 0.20 & 0.40 \\
\hline ANFETAMINAS & & 0.00 & 0.00 \\
\hline CANNABIS & & 0.07 & 0.25 \\
\hline OPIÁCEOS & \multirow{5}{*}{ P.L.D. } & 0.01 & 0.03 \\
\hline COCAÍNA & & 0.02 & 0.08 \\
\hline BENZODIACEPINAS & & 0.05 & 0.19 \\
\hline ANFETAMINAS & & 0.00 & 0.00 \\
\hline CANNABIS & & 0.15 & 0.32 \\
\hline
\end{tabular}


Cuadro 2. VD3: Retención del programa

\begin{tabular}{|c|c|}
\hline \multicolumn{2}{|c|}{ RETENCIÓN EN PROGRAMA } \\
\hline P.L.D. & $100.35 / 40=87,5 \%$ \\
\hline P.M.M. & $100.30 / 40=75 \%$ \\
\hline
\end{tabular}

Cuadro 3. Contraste de diferencias de medidas para las variables relativas a consumo de sustancias (VD1, VD2-a, b, c y d)

\begin{tabular}{|c|c|c|c|c|c|}
\hline VARIABLE & OPIÁCEOS & COCAINA & BENZOD. & ANFETAM. & CANNABIS \\
\hline VD & VD1 & VD2-a & VD2-b & VD2-c & VD2-d \\
\hline \multirow{3}{*}{$\begin{array}{l}\text { Valores } \\
\text { P.L.D. }\end{array}$} & $\mathrm{N}: 35$ & $\mathrm{~N}: 35$ & $\mathrm{~N}: 35$ & $\mathrm{~N}: 35$ & $\mathrm{~N}: 35$ \\
\hline & Media: 0.01 & Media: 0.02 & Media: 0.05 & Media: 0 & Media: 0.15 \\
\hline & $S^{2}: 0.0009$ & $S^{2}: 0.0064$ & $S^{2}: 0.0361$ & $S^{2}: 0$ & $s^{2}: 0.1024$ \\
\hline \multirow{3}{*}{$\begin{array}{l}\text { Valores } \\
\text { P.M.M. }\end{array}$} & $\mathrm{N}: \mathbf{3 0}$ & $\mathrm{N}: 30$ & $\mathrm{~N}: 30$ & $\mathrm{~N}: \mathbf{3 0}$ & $\mathrm{N}: \mathbf{3 0}$ \\
\hline & med 0.09 & med: 0.07 & med: 0.20 & med: 0 & med: 0.07 \\
\hline & $\mathrm{s}^{2}: 0.04$ & $\mathrm{~s}^{2}: 0.04$ & $s^{2}: 0.16$ & $\mathrm{~s}^{2}: 0$ & $\mathrm{~s}^{2}: 0.0625$ \\
\hline $\mathbf{Z}$ & -2.17 & -1.28 & -1.88 & -- & 1.13 \\
\hline P calculada & 0.015 & 0.11 & 0.03 & - & 0.87 \\
\hline$\alpha$ y limites & \multicolumn{5}{|c|}{$0.05 ; p=0.95 ;$ Límites: 0.975 y 0.025} \\
\hline $\mathrm{H}_{0}$ & & Se rechaza & & No procede & Se acepta \\
\hline
\end{tabular}

Cuadro 4. Contraste de diferencias de medias para retención (VD3)

\begin{tabular}{|c|c|}
\hline VARIABLE & RETENCIÓN - VD3 \\
\hline G $^{\circ}$ P.L.D. & N inicial: 40; N Final: 35; Media: $0.875 ; \mathrm{S}^{2}: 0.11$ \\
\hline G० P.M.M. & N Inicial: $40 ;$ N Final: 30; Media: $0.09 ; \mathrm{S}^{2}: 0.04$ \\
\hline Z y P & $-6,420 ; 0,00001$ \\
\hline$\alpha$ y limites & $0.05 ; \mathrm{p}=0.95 ;$ límites: 0.975 y 0.025 \\
\hline $\mathbf{H}_{\mathbf{0}}$ & Se rechaza la $\mathrm{H}_{\mathbf{0}}$ \\
\hline
\end{tabular}


aspectos verdaderamente activos de los paquetes terapéuticos en drogodependencias, no es un problema exclusivo de este trabajo, sino uno de los más habituales en este campo (Echeburúa, 1.996).

Haciendo un análisis global del conjunto de resultados, nos encontramos, antes que nada con que el P.L.D., basado en técnicas de modificación de conducta con apoyo de Naltrexona, tiene éxito para retener a los sujetos en tratamiento, hacer que haya pocos consumos tanto de opiáceos como de otras sustancias y además, esto se produce en, mayor medida con estos sujetos que con los que llevan a cabo un P.M.M.

Podemos afirmar que ambos grupos, el P.M.M. y el P.L.D. disminuyen el consumo de heroína pero no en la misma medida: los sujetos del P.M.M. siguen teniendo nueve veces más positivos que los del P.L.D.

Se justifica el empleo de una droga como la metadona para mantener a los sujetos en tratamiento y para evitar los riesgos asociados al consumo de heroina. Sin embargo, en ambas variables, retención y consumo de heroína, el grupo del P.L.D. obtuvo mejores resultados: Hubo más sujetos que se mantuvieron en tratamiento y menos consumos de heroina en el P.L.D. que el P.M.M.

Una potencial explicación está en la ausencia de terapias psicológicas sistematizadas en los P.M.M. De los centros de P.M.M. estudiados solo el 10\% aplican a todos sus usuarios tratamiento psicológico sistemático. Solo el 36\% de los mismos tenían psicólogo e incluso, no siempre hacian terapia, sino a veces solo seguimiento (Torres Hernández y Garcia Barrachina, 1.997).

Recuérdese que la Naltrexona no crea adicción ni produce hábito, tolerancia, 0 sindrome de abstinencia, luego no es una droga (Ministerio de Sanidad y Consumo, 1.985). Por lo tanto los tratamientos con Naltrexona son tratamientos libres de drogas.

Elizagárate Zabala (1.997) realizó un estudio acerca de la eficacia a largo plazo de los tratamientos que se inician con una desintoxicación con Naltrexona y obtuvo un 60,4\% la retención a los 6 meses y Álvarez González y Del Río (1.998) encuentran un 55\%. Todo parece indicar que la administración de Naltrexona no solo impide el refuerzo positivo de un hipotético consumo de heroína, sino que además altera los niveles de opioides (Ministerio de Sanidad y Consumo, 1.988) y eso disminuye de la probabilidad de recaída. En todo caso el realizar además terapia psicológica mejora sensiblemente los datos de retención (Sardón de Andrés y Ruiz Plagaro, 1.998).

La tasa de retención de nuestro grupo de P.M.M es muy superior a las de muchos programas P.L.D. convencionales. Esta cifra del $75 \%$ es la misma que maneja como tasa de retención anual la Agencia (2004). No obstante la combinación de elementos terapéuticos de nuestro P.L.D. (Naltrexona y técnicas de modificación de conducta) se muestra superior en cuanto a tasa de retención $(87,5 \%)$.

Podemos concluir que los sujetos del P.L.D. recaen menos que los del P.M.M. y que consumen, en general, menos drogas.

En el futuro sería interesante realizar otras investigaciones que completen este estudio, fundamentalmente en dos aspectos: Por un lado, incluir como variables dependientes algunas de tipo psicológico, tales como depresión, ansiedad, estrés, conductas relacionadas con la salud y calidad de vida y por otra parte, estudiar algún P.M.M. en que los sujetos sí reciban tratamiento psicológico sistemático. 


\section{BIBLIOGRAFÍA}

Agencia Antidroga. (1998). Memoria Agencia Antidroga, 1997. Madrid: Consejería de Salud y Servicios Sociales de la Comunidad de Madrid.

Agencia Antidroga. (2004). Memoria agencia antidroga - 2003. Madrid: Consejería de Salud y Servicios Sociales de la Comunidad de Madrid.

Álvarez González, F. J. y del Río, M. C. (1998). Acamprosato, naltrexona y el tratamiento de la dependencia al alcohol. Revista española de drogodependencias. 23, (1, 1-3).

Arce, F. (1997). Respuestas fisiológicas y de autoinforme de adictos a heroína ante situaciones relacionadas y no relacionadas con drogas. Tesis doctoral: U.C.M.

Bedate Villar, J. (1998). Ambiente terapéutico en los programas de mantenimiento con naltrexona. Conferencia pronunciada en el I Seminario sobre naltrexona. Instituto Complutense de Drogodependencias. Madrid.

Bobes-García, J. Fernández Miranda, J. J. González, g. y Portilla, M. P. (1993). Evaluación de los programas de mantenimiento con metadona en Asturias. Ponencia presentada al IV congreso de la sociedad española de toxicomanías. Valencia. Libro de Actas (134-144).

Buzón, J. L. (1995). Situación actual del Sida en España. II Encuentro de Psicología y Drogodependencias. Madrid: C.O.P.

Caballo, B., González, C. y Costa, J. R. (1990). Epidemiología de la infección por VIH; La infección por el virus de la inmunodeficiencia humana. Patogenia, diagnóstico y tratamiento. Madrid: Ministerio de Sanidad y Consumo, Dirección General de Salud Pública. (29-35).

Casas, M. y Salazar, I. (1993). El fenómeno de las recaídas en el marco del proceso terapéutico. En. J. Cadafalch y M. Casas. (Eds.) El paciente heroinómano en el hospital general. Barcelona: CITRAN.

Comas, D. (1993). La asistencia al drogodependiente en España. Génesis, evolución y perspectivas. Proyecto. 5, (8-12).

Cummings, C. Gordon, J. R. y Marlatt, G. A. (1980). Relapse. Prevention and prediction. En W.
R. Miller (Ed.). The addictive behaviors. Treatment of alcoholism, drug abuse, smoking and obesity. Oxford: England Pergamon.

Duro, P. Casas, M. y Colom, M. (1994). Los programas de mantenimiento con metadona en la politica de disminución de daños. Ponencia presentada a las XXI jornadas nacionales de socidrogalcohol. Madrid.

Echeburúa Odriozola, E. (1994). Evaluación y tratamiento de los trastornos adictivos. Madrid: Fundación Universidad Empresa.

Echeburúa Odriozola, E. (1996). Terapia de conducta en la drogadicción. Vitoria: Universidad del País Vasco, Cátedra de Psicologia.

Elizagárate Zabala, E. (1997). Antagonización rápida de opiáceos. Eficacia y seguimiento-seis meses de una muestra de 91 pacientes. Vitoria: Universidad del País Vasco, Departamento. Neurociencias.

Epps, J., Monk, C. Savage, S. y Marlatt, G. A. (1998). Improving credibility of instructions in the balanced placebo design.-misattribution manipulation. Addict behav, 23, (4, 427-435).

Escudero i Torras, M. E. y Ferrer i Pérez, X. (1996). Evolución de los usuarios de un programa de metadona realizado en Barcelona. XII Jornadas Nacionales de Socidrogalcohol. Oviedo. 509-514.

Flynn, P. M., Cradodock, s. G. Luckey, J. W. Hubbard, R. L. y Dunteman, G. H. (1998). Comorbilidad del trastorno de personalidad antisocial y de los trastornos del estado de ánimo en pacientes con tratamiento por dependencia-sustancias psicoactivas. Revista Española Toxicomanías, (14, 28-36).

García, A. y Graña, J. L. (1987). Aspectos psicológicos de las drogodependencias. En P. F. Ramos. A. García. J. L. Graña y D. Comas (eds.). Reinserción social y drogodependencias. Madrid: Asociación para el estudio y promoción del bienestar social.

Girón, S. S. (1996). Estudio de seguimiento de una muestra de heroinómanos a los seis años de iniciado el tratamiento. Tesis doctoral, Universidad de Cádiz,

Grandfield, R. y Cloud, W. (1996). The ele- 
phant that one sees. Natural recovery among middle-class addicts. Journal of drugs iss. 2, (1, 223227).

Graña, J. L. (1994). Conductas adictivas. Teoría, evaluación y tratamiento. Madrid: Debate.

Hunt, W. A., Barnett, 1. W. y Branch, 1. G. (1971). Relapse rates in addiction programs. Journal of clinical psychology. (27, 455-456).

Kelley, p.. (1989). Chemical dependence treatment review guidelines. Gene. Hospit. Psychiatr.,11, $(4,282-287)$.

Kornblit, A. L. y Mendes, A. M. (1990). Recaídas en drogadicción. Factores de riesgo. Revista espanola de drogodependencias. 15, (2, 97-109).

Legarda, J. J. y Gossop, M. (1994). A 24-h impatiens detoxification treatment for heroin addicts.-preliminary investigation. Drug and alcohol dependence, $(35,91-93)$

Llorente del Pozo, J. M. (1997). Validación del DCTQ-H a la población española. Cuestionario de confianza ante situaciones de riesgo de consumo de heroina. Tesis doctoral. Madrid: UNED.

Llorente del Pozo, J. M. Fernández, C. y Vielva, I. (1997). Ansiedad y actividad cognitiva. Un estudio en comunidades terapéuticas para toxicómanos. Leído sin publicar.

Maddux, J. y Desmond, D. (1986). Relapse and recovery in substance abuse careers, NIDA research monograph. (72, 49-71).

Marlatt, A. y Gordon, J. (1980). Determinants of relapse. En P. O. Davidson y S. M. Davidson (Eds.) Behavioral medicine. Changing heath life styles. New York: Brunnel-Mazel.

Marlatt, G. A. (1985-a). Cognitive assessment and intervention procedures for relapse prevention. En G. A. Marlatt y J. Gordon (Eds.). Relapse prevention. New York: Guilford Press.

Marlatt, G. A.(1985-b). Cognitive factors in the relapse process. En G. A. Marlatt y J. Gordon (Eds.). Relapse prevention. Maintenance strategies in the treatment of addictive behaviors. New York: Guilford Press.

Marlatt, G. A.(1985-c). Coping and substance abuse. Implications for research. En S. Shiffman y T. A. Wills (Eds.). Coping and substance use. Orlando; Academic Press.
Marlatt, G. A. (1985-d) lifestyle modification. En G. A. Marlatt y j. Gordon (Eds.). Relapse prevention. New York: The Guilford Press.

Marlatt, G. A. (1985-e). Situational determinants of relapse and skill-training interventions. En g.-Marlatt y J. Gordon (Eds.). Relapse prevention. Maintenance strategies in the treatment of addictive behaviors. New York: The Guilford Press.

Marlatt, G. A.(1985-f). Relapse prevention.-theoretical rationale and overview of the model. En G. A. Marlatt y j. Gordon. (Eds.). Relapse prevention. Maintenance strategies in the treatment of addictive behaviors. Nueva York: The Guilford Press.

Marlatt, G. A.. (1990). Cue exposure and relapse prevention in the treatment of addictive behaviors. Addictive behaviors, (15, 395-399).

Marlatt, G. A.(1992). Substance abuse. Implications of-biopsychosocial model for prevention, treatment, and relapse prevention. En J. Grabowski y G. R. Van den Bos (Eds.). Psychopharmacology. Basic mechanisms and applied intervention. Washington: A.P.A.

Marlatt, G. A. Tucker, J. A. Donovan, D. M. y Vuchinich, R. E. (1997). Help-seeking by substance abusers. The role of harm reduction and behavioral-economic approaches to facilitate treatment entry and retention. NIDA research monograph. $(165,44-84)$.

Martínez Hernández, M. (1993). Metodología aplicada a la investigación en Psicología. Madrid: Editorial Complutense.

Martínez Hernández, M. (1994). Métodos y diseños de investigación en psicologia. Madrid: Editorial Complutense.

Ministerio de Sanidad y Consumo. (1985). Naltrexona clorhidrato. Nuevos productos, $21,(6,257$ 276).

Ministerio de Sanidad y Consumo. (1998). Evaluación de la efectividad del tratamiento "UROD" asociado-rehabilitación ulterior en pacientes dependientes de la heroina. Informe descriptivo. Madrid: Agencia de Evaluación de Tecnologías Sanitarias, Ministerio de sanidad y Consumo.

Nahas, G. Y Trouvé, R. (1992). Manual de toxicomanías. Barcelona: Masson.

Pascual Fernández, C. (1999). Dispensación de 
metadona en el marco de programas de reducción de daños. Evaluación, ventajas e inconvenientes. Congreso europeo sobre rehabilitación y politica de drogas. Marbella. Libro de ponencias, (147-159).

Plan Nacional Sobre Drogas. (1992). Memoria de 1990. Madrid: Mo de Justicia e Interior.

Plan nacional Sobre Drogas. (1998). Observato rio español sobre drogas, informe $n^{\circ}$ 2. Madrid: Delegación del Gobierno para Plan Nacional sobre Drogas.

Plan Regional sobre Drogas. (1992). Memoria. Madrid: Consejería de Integración Social de la Comunidad de Madrid.

Plan Regional sobre Drogas. (1995). Reducción de daños y riesgos asociados al consumo de drogas. Madrid: Consejería de Integración Social de la Comunidad de Madrid.

Rodríguez Molina. J. M. (1995). Estudio acerca de las variables relevantes para predecir éxito en las desintoxicaciones en U.D.H. Madrid: Plan regional Sobre Drogas. Consejería de Integración Social. Comunidad de Madrid.

Rounsaville, B.. Weissman, M. M.. Kleber, H. D. y Wilber, C. (1982). Hetereogeneity of psychiatric diagnosis in treated oppiate addicts. Archives generals of psychiatry, (39, 151-156).

Sardón de Andrés, J. y Ruiz Plagaro, J. A. (1998). Aportación desde el grupo psicoterapéuticola eficiencia en los tratamientos. V Encuentro nacional sobre drogodependencias y su enfoque comunitario. Chiclana de la Frontera (Cádiz), 11 al 13 de marzo. Libro de comunicaciones, (289-292).

Schulteis, g. Y koob, g. F. (1996). Reinforcement processes in opiate addiction.-homeostatic model. Neurochemichal research, 21, (11, 1437-1554)

Self, D. W. y Nestler, E. J. (1998). Relapse to drug-seeking. Neural and molecular mechanisms. Drug and alcohol depend, 51. (1-2, 49-60).

Solé Puig. (1989). Terapia antidroga. Barcelona: Salvat.

Soler Insa, P. A. y Solé Puig. (1981). Clínica de opiáceos. En F. Freixa y P. A. Soler Insa (Eds.). Toxicomanías. Un enfoque multidisciplinario. Barcelona: Fontanella.

Torres Hernández, M. A. y García Barrachina, M. J. (1997). Uso de metadona en España. II Congreso internacional sobre toxicodependencias. Lisboa. Libro de comunicaciones, (168-184). 